\title{
La gestion du régime pédagogique de CEGEPs
}

\author{
JEAN-CLAUDE FORTIN ${ }^{*}$
}

Mis en place il y a bientôt huit ans, les collèges d'enseignement général et professionnel sont l'objet de nombreuses critiques et de fréquentes contestations. Chargés d'assurer à tous les Québécois qui en ont les aptitudes la formation générale en même temps qu'une spécialisation suffisante pour accéder à des études universitaires ou au marché du travail, les CEGEPs ont de moins en moins la faveur des universitaires qui déplorent la spécialisation prématurée et l'absence de rigueur intellectuelle, de formation fondamentale chez un trop grand nombre d'étudiants, pas plus, d'ailleurs, qu'ils n'ont su trouver grâce aux yeux des industriels qui jugent insuffisante la formation spécialisée donnée aux futurs techniciens. Appelé à s'ajuster aux définitions souvent contradictoires des besoins de la société québécoise, confronté quotidiennement avec les aspirations souvent incertaines, indécises de la population étudiante, prisonnier d'un régime pédagogique qui réduit chaque institution au même commun dénominateur, le CEGEP n'a pas encore réussi à se situer comme "niveau d'études complet en lui-même, nettement distinct à la fois du cours secondaire et de l'enseignement supérieur" 1 ; le CEGEP est donc toujours à la recherche de son identité.

Cet article a pour but de présenter les différentes remises en question qu'a connues le régime pédagogique ${ }^{2}$ des CEGEPs au chapitre de l'équilibre entre la formation générale et la spécialisation; de préciser l'influence prépondérante qu'ont eue les universités dans l'élaboration des programmes d'études du niveau collégial et de faire état des intentions nouvelles qu'elles ont récemment manifestées à ce sujet; enfin, de dégager quelques conséquences, sur les programmes d'études, de la gestion du régime pédagogique telle que pratiquée dans les CEGEPs.

Formation générale et spécialisation: les remises en question

Les programmes d'études réalisés dans la première moitié de l'année 1967 respectent les grandes lignes du modèle abstrait proposé par la Commission Parent. La principale difficulté rencontrée fut celle d'assurer, dans les faits, l'équilibre entre la formation générale et la spécialisation. A cet égard, la Commission Parent avait recommandé "que l'on

*Directeur-adjoint des services pédagogiques au Collège Jean-de-Brébeuf, à Montréal.

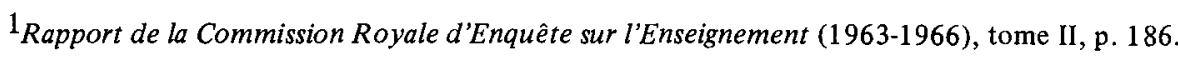

${ }^{2}$ Dans le cadre de cet article, la notion de régime pédagogique est essentiellement réduite aux éléments suivants: "la composition et le contenu des programmes de formation selon des objectifs et des profils"; "la définition des objectifs et des contenus de cours"; "l'agencement des cours et leurs séquences." Conseil supérieur de l'Education, L'activité éducative, Rapport annuel 1969-70, p. 20. 
établisse au départ qu'un tiers du programme de chaque étudiant sera consacré aux cours . communs, un tiers, à des cours spécialisés et un tiers à des cours complémentaires ou connexes." 3 Les exigences des facultés universitaires et du marché du travail eurent cependant pour effet d'accroitre le pourcentage des cours consacrés à la spécialisation; en effet, alors que la Commission avait proposé seize (16) cours de formation générale et huit (8) de spécialisation, le régime pédagogique prévoyait douze (12) cours de formation générale huit (8) cours communs et quatre (4) cours complémentaires) et douze (12) de spécialisation. Par ailleurs, l'article 6 avait pour but de contrer toute velléité de surspécialisation ou de dispersion de l'étudiant en statuant que les douze (12) cours du champ de spécialisation "devaient être choisis dans trois (3) ou quatre (4) disciplines" et qu" "on ne pouvait choisir plus de six (6) cours dans une même discipline." 4 Les cours de spécialisation devaient être choisis dans l'un des trois groupes de disciplines suivants: sciences, sciences humaines et arts et lettres. La promotion par matière constituait une autre garantie de la souplesse du régime en ceci qu'elle offrait la possibilité d'adapter la charge de l'étudiant à ses capacités. Enfin, de nombreux cours optionnels, aussi bien dans le champ de spécialisation qu'en dehors de celui-ci, devaient assurer le respect des goûts et des aptitudes de l'étudiant. Bref, les négociations entre le Ministère, les anciennes facultés des arts, les facultés universitaires et le monde du travail se soldèrent par la rédaction d'un régime pédagogique qui collait assez bien à "la préoccupation d'un système d'enseignement plus riche et plus large, plus souple et plus simple, plus généreux et plus démocratique"5 contenue dans le Rapport Parent.

Par contre, les membres de la Commission Parent avaient jugé bon de faire la mise en garde suivante: "Il ne sera probablement pas facile d'arriver à une formule équilibrée de cours communs, de cours spécialisés et de cours complémentaires. Ce sera d'autant plus difficile que les jeunes adultes de cet âge s'engagent dans des orientations très variées. Beaucoup d'expérimentation et de réflexion seront nécessaires . . ."6 Cette prévision allait s'avérer étonnamment juste car moins de trois ans après la création des premiers CEGEPs on assistait à la première remise en question des programmes d'études de ce niveau d'enseignement.

Le Rapport Roquet: Le CEGEP n'avait pas encore terminé sa première année d'existence que déjà des critiques étaient formulées à l'endroit des cours communs (cours de langue et de littérature anglaise ou française, selon le cas, et cours de philosophie), c'est-à-dire à l'endroit des cours dont la double fonction était d'assurer la formation générale de tous les étudiants et d'opérer un rapprochement culturel entre les étudiants qui se destinent au monde du travail et ceux qui se destinent à l'université. Sans aller jusqu'à recommander la disparition des cours communs, les critiques souhaitaient les voir adaptés aux besoins de la société contemporaine; plus particulièrement, on demandait de mettre l'étudiant en contact avec les principaux univers de connaissance, de tenir davantage compte des

${ }^{3}$ Rapport de la Commission Royale, tome II, p. 168.

${ }^{4}$ Ministère de l'Education du Québec, Cahiers de l'enseignement collégial 1974-75, p. 0-17.

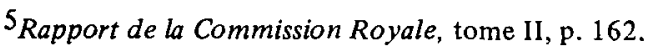

$6_{\text {Ibid., p. 167-168. }}$ 
besoins de la société industrielle, d'assurer une solide introduction aux sciences économiques (trois aspects de la formation que les cours de "Humanities" des CEGEPs anglophones semblaient plus susceptibles d'assurer que les cours de philosophie des CEGEPs francophones) et, enfin, d'accorder une large place à l'amélioration de la communication parlée et écrite dans les cours de français. En réponse à ces critiques, le Comité d'étude des cours communs à tous les étudiants du CEGEP formula 26 recommandations qui, fidèles à l'esprit du Rapport Parent, devaient permettre "de diversifier le contenu de cette formation (générale), d'y introduire un meilleur équilibre, de permettre à l'étudiant d'exercer un choix, de favoriser davantage la rencontre de l'enseignement général et de l'enseignement technique" et dont la principale était la suivante:

En plus du champ de concentration ou de spécialisation, nous recommandons, qu'en vue de la formation générale, tout étudiant soit tenu de prendre des cours dans chacune des catégories suivantes:

- 3 cours de mathématiques et/ou sciences de la nature

- 3 cours de sciences humaines

- 3 cours de philosophie

- 3 cours de langue maternelle et littérature

- 3 cours de technologie

- 2 cours de langue seconde

- 2 cours d'éducation physique.

L'étudiant sera dispensé de prendre les cours de la catégorie qui correspond au champ de concentration ou de spécialisation qu'il a choisi. ${ }^{7}$

Faute d'un consensus sur le contenu de la formation générale qui devait être donnée à tous les étudiants et en raison des réaménagements importants qu'il aurait fallu apporter à la composition du corps professoral de chacun des CEGEPs, la Direction générale de l'enseignement collégial (DIGEC) crut préférable de soumettre les recommandations du Rapport Roquet - du nom de la présidente du Comité - à une étude plus approfondie.

L'hypothèse "C': En mars 1971, un comité composé de cinq Directeurs des services pédagogiques et de deux représentants de la DIGEC se vit donc donner le mandat d'étudier les recommandations du Rapport Roquet dans le but d'en préciser les modalités d'application. Ayant établi que la structure des programmes d'études du niveau collégial devait respecter les principes suivants: "laisser à l'étudiant la possibilité de garder contact avec chacun des grands univers de connaissance sans l'obliger à entrer en contact avec chacun d'eux, cette obligation étant plutôt celle du niveau secondaire," "pour les cours de formation générale, laisser à l'étudiant la possibilité d'exercer un choix des cours" et "reconnaitre la valeur de formation générale des cours du champ de concentration ou du champ de specialisation,"8 les membres du Comité formulèrent trois hypothèses dont l'appellation, assez peu originale, fut " $A$ " - où l'on reprit la structure des programmes d'études pré-

${ }^{7}$ Rapport du Comité d'étude des cours communs à tous les étudiants du CEGEP, ler décembre 1970 , p. 50.

${ }^{8}$ Rapport du Comité des DSP sur le Rapport Roquet, 20 avril 1971, p. 1-2. 
conisée par le Rapport Roquet -, "B" et "C", qui étaient autant d'aménagements de l'hy pothèse " $A$ ". La différence essentielle entre l'hypothèse " $A$ " et l'hypothèse " $C$ ", celle qui sera retenue par le Comité des DSP, résidait dans le fait que cette dernière obligeait tout étudiant, quelque soit son champ de spécialisation, à suivre des cours de sciences, de sciences humaines et d'arts et lettres, ${ }^{9}$ donc dans trois grands secteurs du savoir contrairement aux sept secteurs proposés par le Rapport Roquet.

L'hypothèse " $C$ "' se vit réserver un sort identique à celui du Rapport Roquet: on l'achemina vers les archives. Les raisons du rejet étaient toujours les mêmes: 1) le consensus sur le contenu de la formation générale s'avérait - la chose devenait chaque mois davantage évidente - de plus en plus impossible à réaliser et 2) les réaménagements nécessaires du corps professoral de chacun des CEGEPs, bien que quantitativement moins considérables que ceux qu'auraient provoqués le Rapport Roquet, ne pouvaient pas emporter l'adhésion des professeurs impliqués. Il importe toutefois de noter que certaines recommandations contenues dans le Rapport Roquet et l'hypothèse " $\mathrm{C}$ " furent retenues, non pas sous la forme de règlement ${ }^{10}$, mais dans la pratique; ce fut le cas, notamment, de l'idée de mieux définir les objectifs propres aux cours communs, de celle de développer des cours multidisciplinaires et de celle, enfin, de laisser à l'étudiant plus de choix parmi les cours visant la formation générale. Le personnel des collèges a, de fait, consacré beaucoup d'énergies, au cours des trois dernières années, à la réalisation de ces trois recommandations.

Le "nouveau régime pédagogique": Durant dix-huit mois environ ce fut l'accalmie; les multiples comités de matière s'employaient à ajouter de nouveaux cours à une liste déjà longue, les professeurs à préciser les objectifs propres à chacun des cours offerts et à mettre au point quelques cours multidisciplinaires tandis que les Directeurs des services pédagogiques consacraient une bonne partie de leur temps à la conversion des cours en crédits. En septembre, 1972, l'accalmie fut rompue par la publication d'un projet de régime pédagogique de l'enseignement collégial portant la signature du Directeur du service des programmes et examens. Ce document de travail eut l'effet d'une bombe dans le milieu collégial en raison du fait qu'il avait été préparé dans le plus grand secret par les seuls fonctionnaires de la DIGEC, qu'on a cru à une application rapide et immédiate du projet présenté et, surtout, parce qu'il s'agissait d'un projet de règlement susceptible de rendre définitif, par arrêté en conseil, le régime pédagogique de l'enseignement collégial ... jusque là expérimental et provisoire.

La principale proposition du projet de règlement consistait à réduire de quatre (4) à deux (2) le nombre des cours de philosophie de façon à "laisser plus de latitude à l'étu-

${ }^{9}$ A titre d'exemple, l'étudiant de sciences humaines serait tenu de suivre douze (12) cours dans sa concentration (dont quatre (4) de philosophie), six (6) cours de sciences et six (6) cours d'arts et lettres (dont quatre (4) de langue maternelle).

${ }^{10}$ Il est, à ce sujet, intéressant de noter que le régime pédagogique élaboré en 1967 - et c'est toujours le cas en 1975 - était considéré comme expérimental et provisoire. On estimait à deux ou trois ans la durée de la période d'expérimentation préalable à l'adoption d'un projet de règlement par le lieutenantgouverneur en conseil. 
diant dans le choix de ses cours de formation générale." 11 C'était aux sciences humaines que devait revenir la tâche de combler le vide laissé par la suppression de deux cours de philosophie. Ainsi, alors que les Directeurs des services pédagogiques avaient émis le souhait, au printemps 1968, "que les quatre cours obligatoires de philosophie soient bien des cours de philosophie au sens où on l'entend dans la tradition culturelle francophone," 12 le Directeur général de la DIGEC rétorquait, à l'automne 1972: "Le choix de cours auxquels les étudiants anglophones avaient accès, dans leurs "Humanities," nous proposons de l'étendre aux francophones tout en proposant aux anglophones de bénéficier, eux aussi, d'une véritable initiation à la philosophie."'13 A nouveau, on a pu invoquer l'absence de consensus en regard de la formation générale ainsi que la mise à pied de la moitié des professeurs de philosophie pour faire échec au projet.

Plusieurs autres propositions contenues dans le projet - v.g., le recours possible à la réinscription obligatoire - ou façons de procéder - v.g., l'absence de bilan sur l'expérience des cinq premières années d'opération des CEGEPs - suscitèrent l'opposition de la quasi totalité des agents impliqués. D'ailleurs cette opposition fut à ce point forte qu'elle obligea le Ministère à effectuer un repli stratégique en deux temps: on mit d'abord sur la tablette le projet de règlement et on confia ensuite au Conseil supérieur de l'Education l'étude de l'état (bilan) et des besoins de l'enseignement collégial, étude dont les conclusions seront rendues publiques en juin 1975.

L'approche de Dumont et Rocher: Nous terminerons ce survol du cheminement des idées sur la structure des programmes d'études au niveau collégial en résumant le point de vue de Fernand Dumont et Guy Rocher sur le sujet.

Après avoir signalé que les discussions qui entourent le projet d'un nouveau régime pédagogique ressemblent, à s'y méprendre, à celles qui caractérisent la négociation d'une convention collective de travail, les auteurs affirment que "l'intention originelle qu'avait la Commission Parent, lorsqu'elle a recommandé la création des CEGEPs, a été en partie trahie par la manière dont on l'a réalisée, notamment par l'excès de spécialisation qu'on leur a fait subir."14 En hypertrophiant le "début de spécialisation" souhaité par les auteurs du Rapport, on a ouvert la porte aux "idées de concentration, de départementalisation, de profils de cours pour l'admission à l'université, de pré-requis aux pré-requis des pré-requis, entraînant ainsi le CEGEP loin de l'idée de l'institution polyvalente qu'il devait être et loin du système d'options" avec le résultat que les étudiants, "au secondaire, (ils) doivent déjà faire des choix de cours en fonction de leur orientation future au CEGEP, compte tenu de la faculté à laquelle ils veulent finalement parvenir."15

11 Paré, Léo, "Le régime pédagogique de l'enseignement collégial: présentation du projet de règlement," 25 octobre 1972 , p. 19.

12"Extrait du rapport de la réunion des Directeurs des services pédagogiques tenue le ler mars 1968."

${ }^{13}$ Paré, Op. cit., p. 16.

${ }^{14}$ Dumont, Fernand et Rocher, Guy, L'expérience du CEGEP: urgence d'un bilan in Critère, vol. 8 janvier 1973, p. 12.

$15_{\text {Ibid., p. } 13 .}$ 
Face aux divers éléments qui se conjuguent pour exiger une spécialisation toujours plus rapide et plus poussée des étudiants, Dumont et Rocher proposent une série de mesures susceptibles de donner au régime le coup de barre dont il a un urgent besoin, mesures qui sont les suivantes: substituer à la culture générale une culture fondamentale, c'est-à-dire un savoir critique, transférable, un savoir acquis au contact de l'histoire, de l'expression (langue et littérature), des mathématiques et de la philosophie; donner à l'étudiant, dans les faits, la liberté de choisir son orientation et son programme et, conséquemment, substituer la polyvalence individuelle à la polyvalence institutionnelle largement préconisée à ce jour; procéder à une nécessaire réforme de l'université en lui faisant préalablement accepter que l'école secondaire et le CEGEP n'ont pas comme fonction principale de préparer à l'université.

Non officiel, ce texte est peu susceptible d'aller grossir les archives de la DIGEC mais lorsqu'on se remémore les raisons qui ont fait subir ce sort aux documents présentés précédemment, il y a tout lieu de croire que c'est bien parce qu'il n'est pas officiel que ce texte n'y figure pas en très bonne place!

La présentation des principaux rapports qui ont jalonné le cheminement des idées sur le type d'équilibre qui devrait exister entre les cours de formation générale et les cours de spécialisation au CEGEP ou encore sur le contenu même de la formation générale que le CEGEP devrait assurer à tous les étudiants permet de tirer les conclusions suivantes:

1 la répartition des cours qui composent le programme d'études collégiales est restée la même depuis juin 1967 et le type d'équilibre que cette répartition assure entre la formation générale et la spécialisation est toujours expérimental et provisoire;

2 ce statu quo tient, pour l'essentiel, à l'impossible réalisation d'un consensus autour du contenu de la formation générale et, de façon plus large, à l'incapacité des agents imliqués de définir avec précision les objectifs de ce niveau intermédiaire d'enseignement entre le secondaire et l'université ainsi qu'à la résistance du corps professoral à tout changement de la répartition des cours susceptible de produire des déplacements de personnel;

3 on a constamment postulé, à ce jour, une seule et même répartition, un seul et même contenu des cours de formation générale pour tous les étudiants du niveau collégial;

4 et cela, bien que tous soulignent la nécessité de laisser plus de latitude à l'étudiant dans le choix de ses cours de façon à respecter ses goûts et ses aptitudes.

Il importe maintenant de voir plus en détail comment la diversité des exigences qu'ont eues les universités à l'endroit des étudiants du niveau collégial et l'acuité du problème de coordination des deux niveaux d'enseignement qui en a découlé ne sont pas étrangers aux fréquentes remises en question qu'a connues le régime pédagogique des CEGEPs.

\section{Coordination des enseignements collégial et supérieur}

Le Comité de planification de l'enseignement pré-universitaire et professionnel (Copepp) avait suggéré, en mars 1965, la création d'un comité chargé de "déterminer et d'uniformiser les conditions d'admission aux facultés ou écoles d'enseignement supérieur en termes de pré-requis académiques." L'adoption du règlement 3 - arrêté en conseil du 30 mars 1966 par lequel on créait un nouveau niveau d'enseignement pré-universitaire et profes- 
sionnel - allait conduire le Ministère de l'Education à donner suite à la suggestion du Copepp en procédant à la mise sur pied du Comité mixte; la nécessité d'un tel comité se trouvait d'ailleurs implicitement contenue dans l'article 5 du règlement 3 stipulant que "les études requises pour l'admission en première année d'un cours universitaire sont déterminées par règlement après consultation des universités et des autres établissements d'enseignement reconnus pour les fins du présent article". Le Comité mixte se vit confier un double mandat: préciser les conditions générales d'admission à l'université et définir, pour chacun des programmes universitaires, les cours du niveau collégial jugés préalables à l'admission.

La réalisation de la première partie du mandat se solda par la conclusion, en avril 1967, d'une entente entre le Ministère de l'Education et les universités du Québec, entente dont les principaux points, qui apparaissent chaque année à l'article 9 du régime pédagogique des CEGEPs, sont les suivants:

1 "Les universités du Québec s'engagent à ne pas imposer, pour fin d'admission, aux étudiants qui auront réussi les examens des CEGEPs, l'obligation de subir par la suite d'autres examens de même nature."

2 "Il est convenu que chaque université pourra établir ses propres normes d'admission en regard des résultats obtenus aux examens (passés aux CEGEPs), pourvu qu'elle les ait, au préalable, discuté avec les autres universités et le Ministère de l'Education et qu'elle les ait rendues publiques en temps utile."

3 "Les universités conviennent que les étudiants ne reprendront aucun cours réussi au CEGEP."

4 "Après accord avec le Ministère de l'Education, les universités reconnaitront à leur pleine valeur les cours suivis avec succès aux CEGEPs, si le contenu de ces cours se retrouve au programme universitaire de l'étudiant."16

Le caractère par trop imprécis du deuxième point de cette entente allait favoriser une disparité telle des conditions d'admission entre les universités - et ce plus particulièrement à la suite de la création de l'Université du Québec - que le Ministère décida d'y accorder une attention particulière en constituant un nouveau comité, le Comité de liaison de l'enseignement supérieur et de l'enseignement collégial (Clesec); bien qu'il ait "le pouvoir de faire toute recommandation relative à la coordination des deux niveaux d'enseignement, particulièrement en ce qui concerne la politique de l'admission aux universités," 17 le Clesec s'est vu obligé, jusqu'à maintenant, de consacrer tous ses efforts à la révision des structures d'accueil aux études universitaires de premier cycle.

Des conclusions des travaux du Comité mixte sur les cours de niveau collégial jugés préalables à l'admission dans chacun des programmes universitaires, nous retiendrons un certain nombre d'informations utiles à l'étude du problème de la coordination des deux niveaux d'enseignement.

\footnotetext{
${ }^{16}$ Ministère de l'Education du Québec, Cahiers de l'enseignement collégial 1974-75, p. 1-18.

${ }^{17}$ Ministère de l'Education du Québec, Communiqué: "Création d'un Comité de liaison Enseignement supérieur - Enseignement collégial," 12 novembre 1971, p. 3.
} 
- Les exigences de certaines facultés ou écoles universitaires (arts plastiques: 12 cours, médecine: 12 cours, sciences pures et appliquées: 10 cours, sciences de l'administration: 9 cours) s'avérant supérieures aux huit cours de spécialisation proposés par la Commission Parent, on a choisi de modifier les structures de TOUS les programmes d'études du niveau collégial pour répondre à des besoins particuliers.

- Règle générale, les exigences des facultés de sciences et de certaines écoles professionnelles furent beaucoup plus considérables que celles des facultés de lettres ou de sciences humaines de sorte que, en principe, les contraintes exercées par les universités ne seront pas les mêmes pour tous les étudiants.

- Dans certains cas (tels le droit, la géographie, les sciences de l'administration et les sciences sociales), les exigences des universités anglophones furent soit moins précises soit quantitativement moins considérables que celles des universités francophones.

- Enfin, contrairement à ce qui était prévu à l'article 5 du règlement 3 , les cours requis pour l'admission à tel ou tel programme d'études universitaires n'ont pas été déterminés par règlement mais plutôt par des dispositions administratives renouvelées à chaque année depuis 1967 . Cette absence de règlement n'est évidemment pas étrangère à la diversité de plus en plus grande des exigences de certaines facultés ou écoles d'une université à l'autre et, parfois, au sein d'une même faculté ou école d'une année à l'autre.

Telles sont, brièvement résumées, les principales dispositions qui ont régi la coordination des niveaux collégial et universitaire d'enseignement et qui la régissent d'ailleurs encore aujourd'hui. Telles sont aussi les dispositions qui ont fait dire à plus d'un que le Comité mixte - et plus particulièrement les représentants des universités qui y siègeaient - n'avait pas suffisamment respecté le caractère général de la formation que le CEGEP devait d'abord assurer. Cette accusation peut tenir de l'évidence lorsqu'on sait que les exigences de certaines facultés ou écoles universitaires ont accru de $16 \%$ le nombre de cours de spécialisation du programme d'études collégiales, encore que l'expression "début de spécialisation," à laquelle les membres de la Commission Parent ont toujours eu recours, reste suffisamment vague pour expliquer un écart de $16 \%$ dans un sens ou dans l'autre. D'ailleurs, le problème ne tient pas nécessairement à un nombre précis de cours dont la réussite est préalable à l'admission à la faculté universitaire qui les exige mais bien plutôt à la disparité des exigences des diverses facultés ou écoles d'une même université ou encore à la disparité des exigences d'une même faculté ou école d'une université à l'autre. L'exemple qui suit servira à l'illustrer. L'étudiant qui s'oriente vers le droit n'a pas de cours préalables à réussir; il lui suffit d'obtenir son diplôme d'études collégiales pour être admissible à l'université de son choix. Par contre, si le même étudiant hésite entre le droit et les sciences de l'administration, il devra s'inscrire aux deux cours de mathématiques exigés par les universités anglophones ou, mieux encore, aux quatre cours de mathématiques exigés par les universités francophones s'il veut, à la fin de ses études collégiales, avoir le choix de la faculté et, dans ce cas, de l'université où il compte poursuivre ses études. Lorsqu'on connait le degré d'indécision qui habite un grand nombre d'étudiants à leur entrée au CEGEP, on comprend mieux leur volonté de se soumettre à la structure d'accueil la plus exigente -- en l'occurence celle des sciences - de façon à se laisser le plus grand nombre possible de portes ouvertes lors de leur entrée à l'université. 
Ainsi posé, le problème des structures d'accueil aux études universitaires du premier cycle et, de façon plus générale, le problème de la coordination des niveaux collégial et supérieur d'enseignement semblent ne devoir trouver de solution que dans l'une ou l'autre des deux approches suivantes.

1 “On pourrait regarder l'enseignement collégial (général) uniquement ou avant tout comme le début d'un premier cycle universitaire. L'enseignement général donné au collège serait alors, en fait, de l'enseignement universitaire décentralisé. Les objectifs de l'enseignement collégial (général) seraient alors ceux que s'est donné ou se donnera le premier cycle universitaire. Les programmes d'enseignement général dispensés par les collèges, leur contenu et leur durée, seraient déterminés par les exigences des différents programmes universitaires."

2 "On pourrait regarder l'enseignement collégial (général) comme un cours complet en lui-même. Ce cours pourrait être terminal pour un bon nombre d'étudiants. Il pourrait tout aussi bien permettre une spécialisation ultérieure, soit à l'université, soit en milieu de travail, soit au collège même (en technologie, par exemple). L'enseignement collégial se donnerait alors comme but essentiel la formation fondamentale: épanouissement et maturation du jeune étudiant; initiation aux démarches et aux concepts fondamentaux des disciplines; développement de l'aptitude à penser logiquement, à analyser objectivement, à poser clairement un problème. Les programmes de l'enseignement collégial, libérés des contraintes universitaires, pourraient être révisés en fonction de leurs objectifs propres, favorisant davantage un choix cohérent d'activités de formation, le respect des intérêts de l'étudiant et la formation personnelle."18

Dans la mesure où nous voudrons éviter de nous retrouver dans "la situation paradoxale où la formation de premier cycle va être plus générale que celle donnée au CEGEP,"19 dans la mesure aussi où nous voudrons bien tenir compte des conclusions de plusieurs études récentes (Carnegie, Wright, Roy), la seconde solution s'impose.

Nous entreprendrons maintenant de montrer le chemin qui reste à parcourir aux différents agents impliqués dans l'activité éducative du CEGEP afin d'en faire un lieu de formation fondamentale.

\section{Gestion du régime pédagogique dans les CEGEPs}

Cette dernière partie s'appuie sur notre propre expérience de la gestion des programmes d'études du niveau collégial ainsi que sur les constatations de Jacques Dufresne ${ }^{20}$ et de Jean Proulx ${ }^{21}$ qui viennent, en quelque sorte, la valider; elle vise à mettre en relief certains

${ }^{18}$ Conseil supérieur de l'Education, Etude sur l'enseignement collégial, dossier no 5: Etudiant et Enseignement général, avril 1974, p. 2-3.

${ }^{19}$ Hurtubise, René, L'université québécoise du très proche avenir, Ed. Hurtubise HMH, Montréal 1973, p. 72.

${ }^{20}$ Dufresne, Jacques, Le collège classique et le CEGEP: a struggle for teaching in Le Devoir, samedi, 10 novembre 1973.

${ }^{21}$ Proulx, Jean, Le CEGEP contre l'humain in Maintenant, no 135, avril 1974, p. 24-27. 
aspects de la gestion du régime pédagogique et, surtout, à dégager les effets d'une telle gestion sur les étudiants, les professeurs et les administrateurs des CEGEPs.

La gestion: Au printemps de chaque année scolaire, les étudiants procèdent au choix de leurs cours pour les deux sessions qui suivront. Ce choix est habituellement précédé de sessions d'information sur les programmes et les cours offerts par le collège et il est fait lors d'une rencontre individuelle avec l'un des conseillers aux programmes des services pédagogiques. L'assistance ainsi fournie à l'étudiant est dictée, en quelque sorte, par la réalité même du CEGEP. Ce dernier est, en effet, "un super-marché ou un magasin à rayons. Il a son catalogue qui parait chaque année et qui, en ce qui a trait à la quantité et à la variété des articles qu'il offre, ne le cède en rien à celui des grandes maisons de commerce." 22 S'il est facile pour l'étudiant de s'y retrouver dans le rayon des sciences en raison des séquences rigoureuses que forment ces cours, il en va tout autrement dans les rayons des lettres et des sciences humaines où l'étudiant doit s'en remettre à ses seuls intérêts dans le choix de ses cours. Ceci est d'ailleurs devenu particulièrement évident avec les récentes diminutions des structures d'accueil aux études universitaires; privé du diktat de l'université, l'étudiant, et plus particulièrement celui qui s'inscrit pour la première fois au CEGEP, aura souvent tendance à exiger du conseiller aux programmes qu'il lui dicte les cours à suivre. On veut bien laisser à l'étudiant le plus de latitude possible dans le choix de ses cours, respecter ses goûts, ses aptitudes mais encore faudrait-il que l'étudiant soit capable de trouver en lui-même la détermination, les motivations qui lui permettront de faire les choix demandés.

C'est d'ailleurs sans doute pour accroître les capacités d'expertise et de motivation de l'étudiant qu'on consacre les deux premières semaines de chaque session à ce qu'il convient d'appeler une véritable période de maraudage. L'étudiant a alors le loisir de circuler librement à travers le super-marché et d'y palper la consistance, la force de résistance, bref, la qualité des différents produits qui sont offerts. C'est sans doute ce qui a fait dire à Jacques Dufresne que "le choix des articles, en l'occurrence les cours, est fait selon des rites et des critères qui ne sont pas sans rappeler ceux qui ont cours sur le parquet de la bourse." C'est donc dans un parfait contexte d'économie libérale que la loi de l'offre et de la demande viendra combler le vide laissé par l'absence d'intérêt et de motivation de plusieurs étudiants. On peut déjà entrevoir les effets d'une telle pratique sur l'enseignement et l'apprentissage.

A l'absence de motivation et de points de référence chez bon nombre d'étudiants ainsi qu'au libre jeu de l'offre et de la demande il faut ajouter certaines pratiques administratives qui contribuent à réduire encore davantage la valeur des choix faits par l'étudiant. C'est ainsi que pour assurer une pleine charge d'enseignement aux professeurs qui sont permanents, la plupart des collèges ont recours, sur une échelle plus ou moins grande, à la vente des cours sous pression. Bien sûr, on se retrouve alors à l'antipode du respect des goûts et des aptitudes de l'étudiant mais cette pratique est dictée par le fait que le libre choix de l'étudiant ne concorde pas toujours, peu s'en faut, avec la configuration du corps professoral déjà sur place. On applique donc la technique du "premier arrivé, premier servi" (tout comme au super-marché) tant et aussi longtemps qu'il y a des places disponi-

${ }^{22}$ Dufresne, Op. cit. 
bles dans les différents cours; les étudiants retardataires devront ensuite se contenter de ce qui reste sur les tablettes. Il faut aussi préciser qu'il n'est pas toujours possible de mettre au point un horaire capable de recevoir une multitude de programmes différents. Il en résulte inévitablement des conflits d'horaire qui ont pour effet d'obliger les étudiants touchés à faire le choix de nouveaux cours, lesquels ne sont pas nécessairement conformes aux goûts et aux aptitudes de ces étudiants. Soulignons enfin que, par une sorte de logique implacable, on permet à l'étudiant d'abandonner un ou des cours lorsque le choix s'est avéré inopportun ou encore lorsque les exigences apparaissent . . . trop considérables.

Ses effets: La pratique du libre choix des cours conjuguée à certaines procédures administratives dans l'élaboration des programmes d'études a pour effet, sur le plan pédagogique, d'émietter l'enseignement et de lui enlever à peu près toute cohérence. Emiettement parce que le professeur est soumis à l'éternel recommencement inhérent au fait que des étudiants qui n'ont pas ou peu de connaissances dans telle ou telle discipline cotoient régulièrement, dans le même cours, des étudiants qui ont déjà un certain nombre de connaissances acquises dans un, deux ou même trois cours. Les sciences, nous l'avons déjà souligné, font exception à la règle. Emiettement parce que le professeur n'a pas de prise sur l'étudiant touche - à - tout, sur cet étudiant qui peut faire n'importe quoi, n'importe quand. Il va sans dire, dans ces circonstances, que le niveau collégial a eu tôt fait de perdre les "maitres à penser" d'un autre âge. Pour ce qui est de la cohérence, qu'il suffise de dire que dans la mesure où elle est fort difficile à réaliser, de façon verticale, à l'intérieur d'une même discipline, elle est tout simplement irréalisable, de façon horizontale, entre diverses disciplines. Au niveau de l'enseignement, et il en sera forcément de même au niveau de l'apprentissage, il n'y a pas de programmes d'études cohérents mais plutôt des cours qui se juxtaposent les uns aux autres et s'enchainent à la façon dont les émissions de télévision se succédent.

La pratique du libre choix a aussi donné naissance, dans l'enseignement collégial, à la loi de la jungle. Placés en situation de "sauver leur peau", plusieurs professeurs ont vite compris que dans un super-marché ils avaient tout intérêt à se faire vendeurs! Tous les moyens (attribution de résultats élevés, diminution des exigences académiques, dilution des contenus, etc.) sont donc bons, aux yeux de certains, pour attirer la clientèle. Certains clients ont alors tôt fait d'utiliser le pouvoir qu'une telle situation leur donne pour faire "baisser les prix." Quant au professeur qui décide d'ignorer cette situation, il s'expose à une contestation continue. La concurrence entre les professeurs en amène d'ailleurs certains à abdiquer, soit parce qu'ils en arrivent à la conclusion que ce qu'ils exigent d'euxmêmes et de leurs étudiants appartient à des temps révolus, soit parce qu'ils estiment, en bonne logique, que le "système" l'exige. En somme, certains professeurs, et souvent parmi les meilleurs, ne savent plus trop où ils vont ni où ils doivent aller, ce qu'ils doivent enseigner et comment l'enseigner.

En ce qui a trait aux effets de la gestion des programmes d'études sur la formation des étudiants de niveau collégial, on a beaucoup fait état de l'absence de rigueur dans le raisonnement, de l'éparpillement des connaissances, de la faiblesse de l'expression orale et écrite, bref, de la détérioration de la préparation à l'université. La persistance et l'ampleur de ces critiques nous portent à croire qu'elles sont largement fondées. Il existe cependant un autre effet qui mérite d'être souligné: c'est celui du développement par trop rapide de la 
liberté d'indifférence chez bon nombre d'étudiants. Descartes a défini ce type de liberté comme étant le pouvoir de faire des choix entre divers objets dont aucun n'est ni clairement ni distinctement préférable aux autres et il assigne à la liberté d'indifférence le plus bas degré de liberté. C'est ce type de liberté qui est laissé à l'étudiant du CEGEP dans la mesure où on l'oblige à choisir, à un âge où il est encore très indéterminé, parmi un assortiment de cours digne de notre civilisation d'abondance, ceux qui seront le plus susceptibles de répondre à ses goûts et à ses aptitudes, dans la mesure aussi où "les théories murmurées par les professeurs risquent fort, au lieu de produire l'effet positif libérateur qu'on escomptait, de s'annuler les unes les autres et de créer chez les étudiants plus de confusion que de conviction et plus d'indifférence que de détermination." 23

Enfin, l'administration pédagogique n'échappe pas aux effets de sa propre gestion du régime pédagogique. La description du poste de directeur des services pédagogiques établit, entre autres choses, que ce dernier a pour fonction de planifier, coordonner et contrôler l'activité pédagogique dans l'institution. La coordination faite par les services pédagogiques ne peut cependant être que nominale dans la mesure où les différents cours sont fortement cloisonnés, où on assiste à un chassé-croisé d'étudiants entre tous ces cours, où l'enseignement est départementalisé, bref, dans la mesure où les conditions d'opération tendent beaucoup plus à séparer qu'à unir. Dans de telles conditions, le contrôle est à toute fin utile absent; lorsqu'il existe, il ne porte généralement que sur des détails techniques. Par ailleurs, cette absence de toute coordination efficace et de tout contrôle sérieux, ne permet pas aux services pédagogiques d'assumer le leadership qui leur incombe dans l'institution. Dans la meilleure des hypothèses, on émettra des voeux pieux, on tentera de conserver le peu de dynamisme qui peut encore subsister. Dans la pire, on développera des réflexes de fonctionnaire satisfait d'avoir pu, session après session, assurer à chaque professeur sa juste ration d'étudiants aux termes de la norme $1 / 15$. Quoi qu'il en soit, l'organisation pédagogique en général et la structure des programmes d'études en particulier n'offrent pas aux services pédagogiques les points d'appui nécessaires à l'affirmation de leur leadership. Un bilan (qui est d'ailleurs en voie de réalisation) et des choix s'imposent, de toute évidence.

\section{Conclusion}

Depuis leur création, les CEGEPs sont aux prises avec des problèmes de définition du contenu de la formation générale qu'ils doivent assurer, de coordination avec l'enseignement supérieur et de gestion rationnelle des programmes d'études. Il nous semble que le moment est maintenant venu de relâcher l'étreinte exercée par les universités (les structures d'accueil) et le ministère de l'Education (les articles 4 à 8 du régime pédagogique) sur les CEGEPs, de leur accorder une plus grande autonomie de façon à ce que chaque institution puisse se donner une identité conforme à ses besoins et à ses capacités et, par là, conforme aux besoins et aux capacités des étudiants qui l'auront choisie. Les conclusions du Conseil supérieur de l'Education (étude de l'état et des besoins de l'enseignement collégial) devraient s'avérer déterminantes à cet égard. 